Le monologue intérieur chez Raymond Chandler : révélateur de la société américaine ou piège pour le lecteur?

Pierre-François Peirano

\title{
CpenEdition
}

Journals

Édition électronique

URL : https://journals.openedition.org/ml/5288

DOI : $10.4000 / \mathrm{ml} .5288$

ISSN : 2274-0511

Éditeur

Association Modèles linguistiques

Édition imprimée

Date de publication : 30 décembre 2017

Pagination : 145-164

Référence électronique

Pierre-François Peirano, «Le monologue intérieur chez Raymond Chandler : révélateur de la société américaine ou piège pour le lecteur? », Modèles linguistiques [En ligne], 76 | 2017, document 6, mis en ligne le 10 juin 2019, consulté le 01 juillet 2021. URL : http://journals.openedition.org/ml/5288 ; DOI : https://doi.org/10.4000/ml.5288 


\section{Le monologue intérieur chez Raymond Chandler : révélateur de la société américaine ou piège pour le lecteur?}

\section{Pierre-François Peirano}

Dans son essai "The Simple Art of Murder", publié pour la première fois dans le magazine The Atlantic Monthly, en décembre 1944, le romancier américain Raymond Chandler (1888-1959) tente de définir les ingrédients d'un roman policier réussi et, déjà, plusieurs éléments révèlent ses préférences en matière de narration. Louant le travail de pionnier de Sir Arthur Conan Doyle - dont les enquêtes du personnage de Sherlock Holmes sont relatées à la première personne du singulier par le Docteur Watson -, il reconnaît aussi la valeur des romans d'un autre écrivain américain de l'époque, Dashiell Hammett, l'auteur du Faucon maltais (The Maltese Falcon, 1930), lequel emploie aussi la narration à la première personne du singulier dans deux de ses romans, La Moisson rouge (Red Harvest, 1929) et Sang maudit (The Dain Curse, 1929). Le héros, simplement appelé "The Continental $O p$ ", y relate deux de ses enquêtes sur ce mode. Enfin, le style plus libre utilisé par Hammett est souligné et Chandler en retrace même l'origine au célèbre poète Walt Whitman. Mis bout à bout, ces éléments se révèlent capitaux, lorsqu'il s'agit d'aborder la question du «monologue intérieur» dans les romans de Chandler, car il a appliqué ces principes en mettant l'accent sur la figure du héros - en l'occurrence, le détective privé :

In everything that can be called art there is a quality of redemption. [...] But down these mean streets a man must go who is not himself mean, who is neither tarnished nor afraid. The detective in this kind of story must be such a man. He is the hero, he is everything. He must be a complete man and a common man and yet an unusual man. [...] He must 
be the best man in his world and a good enough man for any world. [... ] He is a relatively poor man, or he would not be a detective at all. He is a common man or he could not go among common people. He has a sense of character, or he would not know his job. [...] The story is his adventure in search of a hidden truth, and it would be no adventure if it did not happen to a man fit for adventure (The Simple Art of Murder, dans Later Novels and other writings : 991-992).

Dans tout ce que l'on peut appeler "art », on trouve un caractère de rédemption. [...] Mais, dans ces rues où règne la mesquinerie, doit passer un homme qui, lui-même, n'est pas mesquin, dont la réputation n'est pas entachée et qui n'a pas peur. Dans ce genre d'histoire, le détective doit être ce genre d'homme. Il est le héros, il est tout dans l'histoire. Il doit avoir tous les attributs de la virilité, doit être un homme du peuple et, cependant, il ne doit pas être comme tout le monde non plus. [...] Il doit être la meilleure personne au monde et un homme assez bon pour vivre dans n'importe quel monde. [...] C'est un homme assez pauvre : autrement, il ne serait pas détective du tout. C'est un homme du peuple; autrement, il ne pourrait pas se mêler au peuple. Il doit savoir ce que signifie avoir du caractère; autrement, il ne connaîtrait pas son métier. [...] L'histoire relate son aventure, sa quête d'une vérité dissimulée, et il ne s'agirait pas d'une aventure, si elle n'advenait pas à quelqu'un qui n'est pas taillé pour cela (traduction personnelle).

Le héros créé par Chandler s'appelle Philip Marlowe et, dans sept romans ${ }^{1}$, il mène les enquêtes qui lui sont confiées à Los Angeles et ses environs. Tous ces romans sont écrits à la première personne du singulier et Marlowe en est le narrateur. Cependant, il est frappant de constater qu'aucune étude approfondie n'a vraiment été faite à ce sujet, car ces sept œuvres constituent autant de monologues intérieurs - voire un seul monologue — fort bien agencés qui, sans donner l'impression « tout-venant » mentionnée par Edouard Dujardin dans sa définition théorique de 1931, entretiennent, néanmoins, d'étroits liens de parenté avec la définition donnée par Valéry Larbaud :

1. Ces sept romans sont, dans l'ordre chronologique : Le grand Sommeil (The Big Sleep, 1939), Adieu, ma jolie (Farewell, My Lovely, 1940), La grande Fenêtre (The High Window, 1942), La Dame du lac (The Lady in the Lake, 1943), La petite Sour (The Little Sister, 1949), The Long Goodbye (publié en 1953 en Grande-Bretagne et en 1954 aux EtatsUnis) et Playback (1958). Quant au roman Marlowe emménage (Poodle Springs, 1959), il n'a pas été achevé par l'auteur. 
Le monologue intérieur est [...] le discours sans auditeur et non prononcé, par lequel un personnage exprime sa pensée la plus intime, la plus proche de l'inconscient, antérieurement à toute organisation logique, c'est-à-dire en son état naissant, par le moyen de phrases directes réduites au minimum syntaxial, de façon à donner l'impression « tout venant $»^{2}$.

Chez Chandler, les fonctions du monologue intérieur sont multiples : à travers le personnage de Marlowe, c'est un tableau complet de la société américaine des années 1940 et 1950 qui prend vie, mais ce choix de narration débouche sur une lecture subjective, voire orientée, et le monologue intérieur confère à Marlowe un statut tout à fait unique, en même temps qu'il sert une vision fort critique de la société, voire de l'être humain. Cet article, dont le but est d'ouvrir des pistes pour des études ultérieures, reprend de nombreux extraits tirés des romans de Chandler sans citer de références purement théoriques et se divise en trois parties. Après s'être plongé dans la pensée même de Philip Marlowe, le regard porté sur la société américaine sera analysé, avant de poser la question de savoir si le monologue intérieur ne constitue pas, dans ces œuvres, un piège pour le lecteur.

\section{Dans la pensée de Philip Marlowe.}

Il convient, tout d'abord, de rappeler que les enquêtes tortueuses relatées dans chaque œuvre sont uniquement décrites à travers le personnage de Marlowe : il n'existe qu'une seule focalisation dans les sept romans et le style, caractérisé par des phrases courtes et incisives, parfois sans verbe, correspond à la définition de Dujardin et traduit les pensées brèves du détective ou son raisonnement par association d'idées. Quant aux paragraphes, ils prennent souvent la forme d'un verset et les comparaisons avec l'œuvre poétique de Walt Whitman, mentionnée par Chandler dans "The Simple Art of Murder", seraient loin d'être illégitimes, même si les intentions du romancier diffèrent de celles du poète. Mais, après ces observations préliminaires, la question suivante - qui s'applique, en fait, à toutes les œuvres écrites sur le mode du monologue intérieur - mérite

2. Édouard Dujardin, Le Monologue intérieur, son apparition, ses origines, sa place dans l'auvre de James Joyce et dans le roman contemporain (1931), Les Lauriers sont coupés (dir. Jean-Pierre Bertrand), GF Flammarion, Paris, 2001 : 136-137. 
d'être posée : à qui Marlowe raconte-t-il ses enquêtes ? Comme le détective n'est pas lui-même écrivain, nous sommes bien en présence d'un "discours sans auditeur et non prononcé », qui mène aux questions bien plus profondes de la création romanesque et de l'utilité d'une œuvre de fiction. Si la question du destinataire reste non résolue, Marlowe semble, tout d'abord, contrôler le cours des événements et le monologue intérieur reflète cet état d'esprit, même lorsque le détective dialogue avec d'autres personnages :

'Then I'm afraid I don't understand what you want, M. Marlowe. What have we to talk about ?' He sounded sly now.

I took the ace out of my sleeve and played it with a languid grace. 'The point is, M. Morningstar, that at the time you called up you already knew the coin wasn't for sale' (The High Window dans Stories and Early Novels : 1010).

- Alors, je crains de ne pas comprendre ce que vous voulez, M. Marlowe. De quoi avons-nous à parler, au juste? Il y avait, maintenant, un petit ton sournois dans sa voix.

Je tire mon as de ma manche et je l'abats avec grâce et langueur.

- Le fait est, M. Morningstar, que vous saviez pertinemment, quand vous avez téléphoné, que cette pièce de monnaie n'était pas à vendre (traduction personnelle).

Cette brève incursion dans la pensée de Marlowe peut s'apparenter à une didascalie, mais il est déjà possible de repérer plusieurs niveaux dans la technique narrative employée. Tout d'abord, le dialogue entre les deux personnages. Ensuite, la perception subjective par Marlowe des intentions de son interlocuteur dans la phrase $\mathrm{He}$ sounded sly now. L'importance de ce dernier mot doit être soulignée, car elle révèle les dons d'observateur de Marlowe, capable de reconnaître un subtil changement de ton. Enfin, Marlowe a recours à une image - il fait donc appel à sa propre subjectivité - pour décrire la réponse qu'il adresse à Morningstar: I took the ace out of my sleeve and played it with a languid grace. La référence au jeu n'est pas innocente : le fait d'abattre une carte gagnante requiert un sens de la stratégie et une réflexion préalable, ce qui va à l'encontre de l'effet « d'immédiateté » recherché dans l'écriture impressionniste. Cette citation illustre déjà la technique d'écriture de Chandler, qui procède par « touches » et utilise aussi de nombreux adjectifs, parfois composés, pour décrire les personnages, alors que la subjectivité propre au monologue 
intérieur se révèle au fur et à mesure. Le lecteur constate ainsi qu'en partant d'une simple observation, le détective donne libre cours à son imagination :

There were two open-grill elevators but only one seemed to be running and that not busy. An old man sat inside it slack-jawed and wateryeyed on a piece of folded burlap on top of a wooden stool. He looked as if he had been sitting there since the Civil War and had come out of that badly (The High Window, op. cit., 1028).

Il y a deux ascenseurs à grille coulissante, mais un seul semble être en service, bien qu'inoccupé pour le moment. A l'intérieur, un vieux bonhomme à la mâchoire pendante et à l'œil humide est assis sur un tabouret en bois recouvert d'un bout de toile à sac pliée. Il a l'air d'être assis là depuis la Guerre de Sécession et d'en être sorti salement amoché (traduction personnelle).

Une véritable progression est à l'œuvre et Marlowe essaie même d'entrer dans les pensées de ses interlocuteurs ou des autres personnages et d'analyser leurs réactions, une technique proche $\mathrm{du}$ « courant de conscience ». Cet emprunt n'est pas rare chez Chandler, qui avait commencé sa carrière en tant que journaliste au Daily Express et avait passé plusieurs années en Grande-Bretagne, mais il y introduit un élément original, dans le sens où le personnage de Marlowe remplace un narrateur omniscient - et extérieur à l'action - dans cette analyse et y associe ensuite ses propres impressions, souvent accompagnées d'une ironie mordante :

Her eyes rounded. She was puzzled. She was thinking. I could see, even on that short acquaintance, that thinking was always going to be a bother to her. (The Big Sleep (1939), Stories and Early Novels, 590).

Ses yeux se mirent à tourner. Elle était perplexe et pensive. Je pus remarquer, même si je la connaissais depuis peu, que penser serait toujours une gêne pour elle (traduction personnelle).

ou, encore, dans l'extrait suivant :

He leaned back again and brooded at me with pale eyes. He fussed around in the chair, trying to get comfortable. A lot of people had tried to get comfortable in that chair. I ought to try it myself sometime. Maybe it was losing business for me (ibid : 1005)

Il se renfonce dans le fauteuil et me couve de son regard pâle et triste. Puis il se tortille pour essayer de trouver une position confortable. J'en ai vu, 
des tas de gens essayer de trouver une position confortable dans ce fauteuil ; il faudrait que j'essaie, moi aussi, un de ces jours. C'est peut-être ainsi que je perdais ma clientèle (traduction personnelle).

La citation précédente est fort révélatrice : en partant de simples constats ou observations, Marlowe donne toujours libre cours à son imagination et passe d'un sujet à l'autre. Il est ainsi facile de constater la transition entre une partie consacrée à la description proprement dite et le moment à partir duquel s'opère le glissement vers la subjectivité. Dans l'extrait suivant, une double barre a été ajoutée pour marquer ladite transition :

At the end of the walk, on a concrete block, there was a little painted Negro in white riding breeches and a green jacket and a red cap. He was holding a whip, and there was an iron hitching ring in the block at his feet. He looked a little sad, as if he had been waiting there a long time and was getting discouraged. I went over and patted his head while I was waiting for somebody to come to the door (ibid : 987)

Au bout de l'allée, sur un socle en ciment, s'érige la statue peinte d'un Noir en tenue d'équitation: culotte blanche, tunique verte et casquette rouge. Il tient un fouet et, à ses pieds, une poignée de fer en forme d'anneau est scellée dans le ciment. // Il a l'air un peu triste de celui qui attend depuis trop longtemps et qui finit par se décourager. Je m'avance vers lui et je lui tapote le crâne, en attendant qu'on se décide à m'accueillir (traduction personnelle).

Par association d'idées ou jeux de supposition, le monologue intérieur se met ainsi en place et peut, tout d'abord, s'articuler suivant une succession logique de pensées, toujours à partir de simples observations :

Minute passed slowly after minute, but I kept on waiting for some new sound. None came. [...]

I looked across to the lighted beach club. From its upper windows a man with a good night glass could probably cover this spot fairly well. He could see a car come and go, see who got out of it, whether there was a group of men or just one. Sitting in a dark room with a good night glass you can see a lot more detail than you would think possible (Farewell, My Lovely, Stories and Early Novels : 811).

Les minutes s'égrenaient lentement, interminables, mais je restais là, debout, à l'affut d'un nouveau bruit. Rien ne vint. [...]

Je portai mon regard de l'autre côté, sur la plage, vers les lumières du club. 
Des fenêtres du haut, un homme muni d'une bonne lunette pour voir la nuit devait pouvoir couvrir l'endroit où je me trouvais. Il pouvait suivre les allées et venues d'une voiture et voir qui en sortait, un groupe d'hommes ou un seul. Assis dans une pièce obscure, avec une bonne lunette, on peut voir bien plus de détails qu'on pourrait le penser (traduction personnelle).

Mais, d'autres fois, Marlowe brosse le portrait complet d'un personnage ou de ses relations - comme, par exemple, dans le passage suivant, à partir de la simple lecture de la carte de visite d'un psychiatre, sur laquelle plusieurs mots commencent par une majuscule. La transition entre la lecture proprement dite et le début du monologue intérieur est, encore une fois, marquée par une double barre ajoutée par l'auteur de l'article. Mais le détective en vient à imaginer le type de patients que ce psychiatre reçoit et donne la parole à ces derniers au moyen du style indirect libre - sans, toutefois, que la ponctuation l'indique, technique proche de celle employée par William Faulkner. Les phrases en question sont en italiques :

Jules Amthor, Psychic Consultant. Consultations By Appointment Only. // Give him enough time and pay him enough money and he'll cure anything from a jaded husband to a grasshopper plague. He would be an expert in frustrated love affairs, women who slept alone and didn't like it, wandering boys and girls who didn't write home, sell the property now or hold it for another year, will this part hurt me with my public or make me seem more versatile? Men would sneak in on him too, big strong guys that roared like lions around their offices and were all cold mush under their vests. But mostly it would be women, fat women that panted and thin women that burned, old women that dreamed and young women that thought they might have Electra complexes, women of all sizes, shapes and ages, but with one thing in common - money. No Thursdays at the County Hospital for Mr. Jules Amthor. Cash on the line for his (ibid : 842)

Jules Amthor, Expert en Psychiatrie. Consultations Sur Rendez-Vous Uniquement. // Donnez-lui assez de temps et d'argent, il vous débarrassera de tous vos maux, depuis le mari fatigué jusqu'à une invasion de sauterelles. Sûrement un expert en matière d'amours contrariées, de femmes qui dorment seules et qui n'aiment pas ça, de parents sans nouvelles de leur fille ou de leur garçon, partis à l'aventure. Faut-il vendre la propriété maintenant ou attendre encore un an? Ce rôle me nuira-t-il auprès de mon public ou, au contraire, montrera-t-il un nouvel aspect de mon talent? Il devait aussi recevoir la visite discrète de nombreux hommes, des grands costauds qui rugissaient comme des lions dans leurs bureaux, mais cachaient une âme en charpie sous leur gilet. Mais, surtout, des 
femmes : de grosses femmes à bout de souffle, des femmes maigres et consumées, de vieilles femmes qui se prenaient à rêver, de jeunes femmes obsédées à l'idée de faire un complexe d'Electre, des femmes de toutes sortes, de toutes tailles et de tous âges, mais qui ont toutes un point commun : pas de consultation gratuite à l'hôpital du comté pour M. Jules Amthor! Il lui faut des espèces sonnantes et trébuchantes (traduction personnelle).

Ce passage constitue l'un des meilleurs exemples de la place réservée par Chandler au monologue intérieur et ce mode de narration sous-tend, en fait, l'intégralité des romans dont Marlowe est le personnage principal. En effet, l'utilisation unique de la focalisation interne est synonyme d'une conception très élastique du temps, contracté ou dilaté au fur et à mesure que l'enquête progresse, et donne lieu à des ellipses, lorsque Marlowe est pris dans ses propres réflexions, s'endort, puis se réveille... ou se fait assommer par un agresseur indélicat. Un chapitre d'Adieu, ma jolie se termine ainsi, puis le détective recouvre ses sens au début du chapitre suivant:

Whoever it was had a nice easy shot at the back of my head. Afterwards I thought I might have heard the swish of a sap. Maybe you always think that - afterwards. [fin de chapitre].

'Four minutes,' the voice said. 'Five, possibly six. They must have moved quick and quiet. He didn't let out a yell.' I opened my eyes and looked fuzzily at a cold star. I was lying on my back. I felt sick (ibid. : 811-812).

Peu importe qui était assis à l'arrière, j'étais idéalement placé pour en prendre un bon coup sur la caboche. Par la suite, je crus me rappeler comme le vague sifflement d'une matraque, mais c'est peut-être une réflexion que l'on se fait toujours - après coup. [Fin du chapitre]

- Quatre minutes, fit la voix. Ou cinq, six au maximum. Ils ont dîu faire vite, et sans bruit. Il n'a même pas laissé échapper un cri.

J'ouvris les yeux et mon regard, comme enveloppé dans du coton, rencontra l'éclat tranchant d'une étoile. J'étais couché sur le dos. J'avais mal au cour (traducton personnelle).

Un procédé similaire est à l'œuvre, lorsque, dans La Dame du lac, Marlowe raconte l'un de ses rêves, puis son réveil. Les liens avec l'un des événements de l'enquête qu'il mène - la découverte du cadavre d'une femme dans un lac - et le caractère macabre du songe, qui plonge également le lecteur dans un profond malaise, doivent être soulignés : 
I dreamed I was far down in the depths of icy green water with a corpse under my arm. The corpse had long blond hair that kept floating around in front of my face. An enormous fish with bulging eyes and a bloated body and scales [...] swam around leering like an elderly roué. Just as I was about to burst from the lack of air, the corpse came alive under my arm and got away from me and then I was fighting with the fish and the corpse was rolling over and over in the water, spinning its long hair.

I woke up with a mouth full of sheet and both hands hooked on the head-frame of the bed and pulling hard. The muscles ached when I let go and lowered them (The Lady in the Lake (1943), Later Novels and Other Writings : 75.

Je rêvais que j'étais perdu dans les profondeurs glacées d'une eau verte, avec un cadavre sous le bras. Il avait de longs cheveux blonds qui continuaient à flotter devant mon visage. Un énorme poisson, avec des yeux saillants, un corps tout enflé et des écailles protubérantes, [...] me tournait autour en me faisant de l'ail comme un vieux pervers. Au moment où j'allais étouffer par manque d'air, le cadavre revint à la vie sous mon bras et s'éloigna de moi, puis je me battais avec le poisson et le cadavre roulait dans l'eau de-ci, de-là, tourbillonnant dans sa longue chevelure.

Je m'éveillai la bouche pâteuse et les deux mains cramponnées aux barreaux de mon lit, tirant dessus de toutes mes forces. Les muscles de mes bras me firent mal, quand je lâchai prise (traduction personnelle).

Les deux précédents exemples sont autant d'illustrations des multiples usages du monologue intérieur par Raymond Chandler, qui utilise ce mode avec un art consommé de la narration. De cette manière, l'enquête avance au fil des pensées, des interrogations et des actions de Philip Marlowe, mais ils ne peuvent être séparés de leur cadre : la société américaine des années 1940 et 1950 - plus, précisément, la ville de Los Angeles et ses environs, dans l'Etat de Californie -, sur laquelle Marlowe porte un regard des plus acerbes.

\section{Un regard critique sur la société américaine}

Cette caractéristique est indissociable des enquêtes menées et n'est jamais gratuite. Au contraire, ce sont les plongées de Marlowe dans des univers équivoques et corrompus qui lui font porter un tel jugement sur ses contemporains et aucune couche de la société n'est épargnée. A de multiples reprises, les idées se bousculent dans l'esprit du détective, mais restent sous-tendues par un cheminement logique, admira- 
blement rendu par le monologue intérieur. Les romans fourmillent de multiples détails et les descriptions ou les successions d'actions révèlent toutes la société de l'époque, comme autant d'instantanés pris sur le vif. Une juxtaposition de plusieurs voix s'opère à travers la narration et la polyphonie correspond ainsi aux actions de Marlowe, aux détails qu'il remarque ou aux bruits de fond qu'il entend :

I smeared the doorknob opening the door and the outside knob closing the door. The Dodgers ${ }^{3}$ were ahead seven to three, the first half of the eighth. A lady who sounded well on with her drinking was singing Frankie and Johnny, the roundhouse version, in a voice that even whiskey had failed to improve. A deep man's voice growled at her to shut up and she kept on singing and there was a hard quick movement across the floor and a smack and a yelp and she stopped singing and the baseball game went right on (The High Window, op. cit. : 1039).

Je prends bien soin d'effacer les empreintes sur les boutons de porte en m'en allant, à l'intérieur comme à l'extérieur. Les Dodgersmènent sept à trois, première mi-temps de la huitième manche. Une femme qui, à l'entendre, semble fortement imbibée, chante "Frankie and Johnny", la version longue, d'une voix que même le whisky ne parvient pas à rendre plus agréable. Une grosse voix d'homme lui intime de la boucler et, comme elle continue de chanter, j'entends quelques pas rapides, un claquement sec, un cri comme un jappement, après quoi, elle arrête de chanter et le base-ball continue (traduction personnelle).

La transition entre les actions de Marlowe et les bruits à l'intérieur d'un appartement voisin n'est, encore une fois, marquée par aucun signe de ponctuation. Il incombe donc au lecteur d'établir la différence entre ces différentes voix, tandis que la dispute qui oppose une femme - probablement malheureuse au point de sombrer dans l'alcool - à son brutal de mari révèle déjà une peinture sans concession de la société, sur fond de commentaires radiophoniques et de "Frankie and Johnny", une célèbre chanson populaire américaine qui se décline en plusieurs versions.

Ainsi, Los Angeles et ses quartiers deviennent même des personnages à part entière et Marlowe se peint souvent comme un élément dans ce vaste décor, non sans une certaine ironie envers lui-même :

Another day drawing to its end, the air dull and tired, the heavy growl of homing traffic on the boulevard, and Marlowe in his office nibbling a drink and sorting the day's mail (ibid : 1126).

3. Célèbre équipe américaine de base-ball, basée à l'époque à Brooklyn. 
Encore une journée qui tire à sa fin : il y avait de la morosité et une certaine lassitude dans l'air. Dehors, le grondement sourd de la circulation, plus dense à cette heure, alors que l'on rentre chez soi. Et, dans son bureau, Marlowe sirotant un verre et rangeant son courrier du jour (traduction personnelle).

Par le biais du monologue intérieur, la vision critique de la société n'est jamais absente, qu'il s'agisse des quartiers pauvres du centre de la ville, comme dans une citation précédente, ou les quartiers résidentiels de la périphérie ou des hauteurs alentour. La technique employée reste la même et, à partir de simples descriptions, Marlowe laisse libre cours à son imagination :

It was close to the ocean and you could feel the ocean in the air but you couldn't see water from the front of the place. Aster Drive had a long smooth curve there and the houses on the inland side were just nice houses, but on the canyon side they were great silent estates, with twelve foot walls and wrought iron gates and ornamental hedges; and inside, if you could get inside, a special brand of sunshine, very quiet, put up in noise-proof containers just for the upper classes (Farewell, My Lovely, op. cit. : 854).

[Ce quartier] était situé à proximité de l'océan. On ne le voyait pas, mais l'air était saturé d'effluves marines. Aster Drive décrivait une longue et large courbe à cet endroit ; les maisons qui la bordaient, du côté de la terre, étaient simplement d'agréables villas, mais celles qui dominaient le canyon étaient de grandes propriétés tranquilles, entourées de murailles de quatre mètres, avec des grilles en fer forgé et, en guise de décoration, des haies. A l'intérieur - mais encore fallait-il pouvoir y pénétrer -, brillait un soleil de premier choix, livraison ultra-silencieuse garantie en container, modèle spécial pour les nantis (traduction personnelle).

Ces descriptions vivantes et ciselées constituent le reflet d'une société émergeant à peine de la Grande Dépression ${ }^{4}$ et toujours marquée par la pauvreté, même pendant la période de prospérité qui a suivi la Deuxième Guerre Mondiale. À travers Marlowe, le constat est cruel : elle reste bâtie sur les apparences, les illusions et la cor-

4. Aujourd'hui, les historiens s'accordent pour dire que la politique du New Deal, mise en place par Franklin D. Roosevelt dès son accession à la présidence, en 1933, n'a pas été suivie de résultats immédiats. Il fallut attendre plusieurs années, même un «Second New Deal », en 1937, pour que les Etats-Unis sortent enfin de la Grande Dépression. Après 1941 et l'entrée en guerre des Etats-Unis aux côtés des Alliés, l'effort de guerre fut synonyme d'une reprise durable de l'économie américaine. 
ruption. A de nombreuses reprises, Marlowe emploie un ton désabusé, parfois nostalgique, pour décrire la ville où il réside et mène ses enquêtes, et le roman La petite Scuur (The Little Sister, 1949) en est sans doute la meilleure illustration, car il révèle la face cachée de Hollywood et du monde du cinéma, où l'arrivisme le dispute à la dépravation. Par effet de miroir, Marlowe décrit un Los Angeles artificiel, en proie à l'urbanisation galopante et au crime organisé, ville dans laquelle le sentiment d'aliénation se fait plus que jamais sentir. Il est à noter que, dans l'extrait suivant, Marlowe emploie le discours direct, tout en laissant dériver ses pensées. On y retrouve l'impression de « tout venant » chère à Edouard Dujardin et le monologue intérieur s'immisce jusque dans les conversations, tout comme le sentiment d'aliénation dans les périphéries de la ville :'

'I used to like this town,' I said, just to be saying something and not to be thinking too hard. 'A long time ago. There were trees along Wilshire Boulevard. Beverly Hills was a country town. Westwood was bare hills and lots offering at eleven hundred dollars and no takers. Hollywood was a bunch of frame houses on the interurban line. Los Angeles was just a big dry sunny place with ugly homes and no style, but goodhearted and peaceful. It had the climate they just yap about now. People used to sleep out on porches. Little groups who thought they were intellectual used to call it the Athens of America. It wasn't that, but it wasn't a neon-lighted slum either. [...] Out in the fancy suburbs dear old Dad is reading the sports page in front of a picture window, with his shoes off, thinking he is high class because he has a three-car garage. Mom is in front of her princess dresser trying to paint the suitcases out from under her eyes. And Junior is clamped onto the telephone calling up a succession of high school girls that talk pigeon English and carry contraceptives in their make-up kit' (The Little Sister (1949, Later Novels and Other Writings : 357-358.

- Auparavant, j'aimais cette ville, dis-je, juste pour essayer de dire quelque chose et ne pas avoir l'air de trop réfléchir. Mais c'était il y a longtemps. Wilshire Boulevard était bordé d'arbres, Beverly Hills était une bourgade rurale. À Westwood, il n'y avait que des collines sans habitation, des parcelles de terrain à onze mille dollars, mais aucun acquéreur ne pointait le bout de son nez. Hollywood n'était qu'un agglomérat de maisons à charpente sur la ligne interurbaine. Los Angeles était une grande ville ensoleillée, aride, sans aucun style; les maisons étaient assez laides, mais l'atmosphère paisible et bon enfant. On y trouvait le climat dont ils ne cessent de jacasser à présent. À l'époque, les gens dormaient 
dehors, sous les porches, de petits groupes qui se disaient intellectuels l'appelaient "l'Athènes de l'Amérique ». Ce n'était pas exactement ça, mais ce n'était pas non plus un taudis illuminé par des tubes au néon. [...] Dans les banlieues chics, on voit Papa qui lit la page des sports devant une fenêtre panoramique: il a enlevé ses chaussures et pense qu'il appartient à la classe supérieure, juste parce qu'il possède un garage assez grand pour trois voitures. Maman est assise devant sa coiffeuse de princesse : elle essaie de faire disparaître les valises sous ses yeux avec une couche de maquillage, pendant que le fiston est rivé au téléphone, appelle sans cesse des jeunes filles qui vont au lycée, parlent un anglais argotique et gardent des contraceptifs dans leur trousse de maquillage (traduction personnelle).

Chandler explore encore plus profondément toutes les possibilités du monologue intérieur et y ajoute même un autre aspect : après avoir laissé dériver son imagination en partant d'une simple succession d'actions, Marlowe fait soudain preuve de recul et juge les propos qu'il vient de prononcer dans son esprit, comme s'il prenait soudainement conscience d'avoir émis un jugement trop sévère. Ces différentes étapes, dans lesquelles le style indirect libre est également utilisé - il imagine les paroles prononcées par le serveur d'un restaurant - sont, encore une fois, privées de toute ponctuation et, au cours de ce monologue, Marlowe égratigne encore Los Angeles et la société américaine dans son ensemble. Les doubles barres qui ont été rajoutées correspondent aux étapes précédemment mentionnées :

I ate dinner at a place near Thousand Oaks. Bad but quick. Feed 'em and throw 'em out. Lots of business // We can't bother with you sitting over your second cup of coffee, mister. You're using money space. See those people over there behind the rope? They want to eat. Anyway they think they have to. God knows why they want to eat here. They could do better home out of a can. They're just restless. Like you. They have to get the car out and go somewhere // Sucker-bait for the racketeers that have taken over the restaurants // Here we go again. You're not human tonight, Marlowe. [...] No moon, no fuss, hardly a sound of the surf. No smell. None of the harsh wild smell of the sea. A California ocean // California, the department-store state. The most of everything and the best of nothing // Here we go again. You're not human tonight, Marlowe (ibid : 268).

Je suis allé dîner à un endroit près de Thousand Oaks. Nourriture de mauvaise qualité, mais service rapide. On leur donne à manger et on les met dehors. Ils sont débordés. // Vous comprenez, Monsieur, on ne peut pas attendre que 
vous restiez assis, là, à finir votre deuxième tasse de café, l'espace que vous utilisez doit être rentabilisé. Vous voyez tous ces gens qui attendent derrière la corde? Ils veulent manger. De toutes façons, ils pensent qu'ils doivent manger. Dieu sait pourquoi ils veulent manger ici. Ils seraient mieux chez eux à ouvrir une boite de conserve. Tout simplement, ils ne tiennent pas en place, comme vous. Il faut qu'ils sortent la voiture et qu'ils aillent quelque part. // Des gogos qui filent leur argent aux trafiquants qui se sont tournés vers la restauration. // Ça y est, ça recommence. Décidément, mon vieux Marlowe, tu n'es pas très humain, ce soir. [...].

Une nuit sans lune, très calme: on entendait à peine le bruit des vagues. On ne sentait rien, absolument rien, même pas l'odeur forte de la mer et du grand large. Juste l'océan de Californie. // La Californie, l'Etat-supermarché : on y trouve la plupart des choses, mais rien de ce qui se fait de mieux. // Ça y est, ça recommence. Décidément, mon vieux Marlowe, tu n'es pas très humain, ce soir (traduction personnelle).

À la lecture des romans, Marlowe fait ainsi office de "décodeur », car il semble révéler le revers de la société américaine de son temps et le mode de narration choisi donne forcément au lecteur le sentiment qu'il (Marlowe) a raison. De cette manière, son caractère profondément désabusé - sans qu'il en devienne malhonnête pour autant - est progressivement révélé, par touches successives. Mais il est cependant légitime de se demander si, au contraire, la vision totalement subjective induite par le monologue intérieur n'exerce pas une influence sur le lecteur, au point d'orienter, voire de fausser son jugement.

\section{Le monologue intérieur : un piège pour le lecteur?}

Le personnage de Marlowe se situe ainsi dans un entre-deux, car il fait tampon entre le lecteur, d'une part, et les péripéties de ses enquêtes et le cadre dans lequel elles se déroulent, d'autre part. Mais, dans cette perspective, le monologue intérieur n'est-il pas un piège, en raison même de l'extrême subjectivité de ce mode de narration ? Le jugement du détective peut se révéler à double tranchant : certes, il est possible de ressentir de l'empathie vis-à-vis de certains personnages, mais l'opinion du lecteur est forcément orientée. Les observations du narrateur à la première personne et le jugement implicite rendu par le monologue intérieur sont à l'origine de ce prisme si particulier, que l'on remarque tout d'abord dans de nombreuses descriptions : 
There she was. She didn't have to open her mouth for me to know who she was. And nobody ever looked less like Lady Macbeth. [...] She had no make-up, no lipstick and no jewelry. The rimless glasses gave her that librarian's look (ibid : 205).

Elle était là. Elle n'avait même pas besoin d'ouvrir la bouche pour que je sache qui elle était. Personne n'avait jamais moins ressemblé à Lady Macbeth. [...] Elle n'était pas maquillée, ne portait ni rouge à lèvres, ni bijoux. Ses lunettes n'avaient pas de monture et lui donnaient l'air d'une bibliothécaire (traduction personnelle).

Marlowe est, en outre, le seul juge des qualités potentielles des autres personnes et aucun autre point de vue ne vient le contredire :

A man named Nulty got the case, a lean-jawed sourpuss with long yellow hands which he kept folded over his kneecaps most of the time he talked to me. [...] He looked poor enough to be honest, but he didn't look like a man who could deal with Moose Malloy (Farewell, My Lovely, op. cit. : 776-777)

L'affaire fut confiée à un nommé Nulty, un vieux grincheux à la mâchoire maigre. Pendant presque toute la conversation, il garda ses longues mains jaunes croisées sur les genoux. [...] Il semblait assez pauvre pour être honnête, mais ne paraissait pas de taille à se mesurer à Moose Malloy (traduction personnelle).

Il s'érige, enfin, en juge du bien et du mal, ainsi que des actions de ses congénères. La citation suivante, au discours direct, s'apparente de nouveau au monologue intérieur et c'est une lecture purement subjective des événements et des personnages qui est proposée au lecteur, même si Marlowe parvient à dénouer les fils de l'enquête, un élément qui ajoute à la légitimité de son jugement :

'I'm not going to turn you in, if that's what you mean. Beyond that I guarantee nothing. If I'm involved in it, I'll have to face up to the situation. There's no question of morality involved. I'm not a cop nor a common informer nor an officer of the court. You say it was an accident. Okay, it was an accident. I wasn't a witness. I haven't any proof either way. I've been working for your mother and whatever right to my silence that gives her, she can have. I don't like her, I don't like you, I don't like this house. I didn't particularly like your wife. But I like Merle. She's kind of silly and morbid, but she's kind of sweet too. And I know what has been done to her in this damn family for the past eight years' (The High Window, op. cit. : 1169). 
- Je ne vais pas vous dénoncer, si c'est ce que vous voulez dire. A part ça, je ne promets rien. Si je me trouve entraîné dans cette histoire, il faudra que je me défende. La morale n'intervient pas là-dedans. Je ne suis ni un flic, ni un mouchard, ni un magistrat. Vous dites qu'il s'agit d'un accident. Bon, d'accord, c'est un accident. Je n'étais pas présent et je n'ai aucune preuve, dans un sens ou dans l'autre. Je travaillais pour votre mère. Dans la mesure où je dois la couvrir par mon silence, je le ferai. Je ne l'aime pas. Je ne vous aime pas. Je n'aime pas cette maison. Je n'aimais pas beaucoup votre femme. Mais j'aime beaucoup Merle. Elle est un peu sotte et maladive, mais elle a un côté adorable. Et je sais par quoi elle est passée, dans cette foutue famille, depuis huit ans'(traduction personnelle).

Cependant, les raisons qui poussent chaque personnage à agir et leurs actions respectives sont ainsi expliquées, même à partir de l'opinion du narrateur. L'image de la société ainsi façonnée est loin d'être manichéenne et, à la lumière des précédentes citations, il s'agit davantage d'un agrégat d'individus aliénés au sein d'une société qui les broie. Le regard sans concession porté par Chandler sur la société de son temps rejoint ainsi le point de vue de Philip Marlowe et, dans plusieurs de ses lettres, l'écrivain s'était souvent assimilé au détective qu'il avait créé : «P. Marlowe and I do not despise the upper classes because they take baths and have money; we despise them because they are phoney " ( $P$. Marlowe et moi, nous ne méprisons pas les bourgeois parce quils prennent des bains et qu'ils ont de l'argent; nous les méprisons, parce que ce sont des faiseurs (traduction personnelle). Il établit, dans cette même lettre, une différence fondamentale entre conscience sociale et conscience personnelle ${ }^{5}, l^{\prime}$ 'origine même de la peinture acerbe d'une société dans laquelle les intérêts et les instincts des individus ne s'accordent pas avec la nécessité d'une vision commune et convenue - au détriment de la morale et, parfois, de la vérité, car le détective peut se trouver contraint de ne pas livrer le véritable coupable, même s'il le connaît, au risque de révéler un scandale plus grand encore. Il garde néanmoins sa propre dignité et c'est à lui, personnage somme toute artificiel, qu'il incombe de révéler l'artificialité de l'organisation et des codes de la société. L'hypothèse selon laquelle l'intrigue ne serait qu'un simple prétexte pour illustrer la philosophie de vie de Marlowe - et, indirectement

5. "P. Marlowe has as much social conscience as a horse. He has a personal conscience, which is an entirely different matter." Lettre à Dale Warren, op. cit., 1023. 
de Chandler - mérite d'être soulevée. Le caractère moralisateur des jugements du détective ne saurait être éludé, comme l'a écrit Charles Trueheart :

[Marlowe] est capable de réprimander certaines personnes pour leurs mauvaises manières, de capituler, lorsque quelqu'un lui raconte ses malheurs, ou de voir le cœur tendre qui se cache derrière les habits les plus agressifs. C'est un croisé solitaire - un 'chevalier des temps modernes', selon la formulation critique consacrée - au milieu d'un univers gangréné par la corruption. En tant qu'arbitre du bien et du mal, il est, au pire, sur un pied d'égalité avec les officiers de la loi dûment nommés.

Il est ainsi possible de rapprocher Chandler d'auteurs tels qu'Anthony Trollope ou Henry James, voire Henry Fielding. Le monologue intérieur rejoint ainsi le débat entre réalité et illusion : ce mode de narration reste un miroir qui peut se révéler déformant et mène à une vision relativiste des autres personnages et de la société dans son ensemble - sans que le bon sens de Philip Marlowe soit remis en question.

Ce dernier use même de l'illusion avec un certain humour. Au premier chapitre du Grand Sommeil (The Big Sleep, 1939), le premier roman dans lequel il apparaît, il commence par donner une identité fantaisiste à l'une des filles de son client, le général Sternwood, sans que le lecteur connaisse encore son véritable nom, prononcé pour la première fois par Norris, le majordome de la maison, quelques lignes plus bas :

'What's your name?

'Reilly,' I said. 'Doghouse Reilly.'

'That's a funny name.' She bit her lip and turned her head a little. [...]

The butler said tonelessly: ‘The General will see you now, Mr. Marlowe'

(The Big Sleep, op. cit.: 590-591).

- Comment vous appelez-vous?

- Reilly, lui dis-je. Doghouse Reilly.

- C'est amusant, comme nom. Elle se mordit la lèvre et tourna légèrement la tête. [...]

Le majordome annonça, d'une voix blanche:

- Le général va vous recevoir, Monsieur Marlow (traduction personnelle). 
Enfin, la narration sur le mode potentiel, quintessence du monologue intérieur, est utilisée à plusieurs reprises, comme dans cet extrait de La grande Fenêtre (The High Window, 1942). A cet instant, Marlowe vient de découvrir un autre cadavre et s'interroge sur les décisions les plus opportunes à prendre en ce moment critique. Il imagine la possibilité d'une inculpation et, une nouvelle fois, révèle le fil de ses pensées de la manière la plus spontanée :

I went back to the living room and stood in the middle of the floor breathing with my mouth as far as possible and wondering what the score would be when I turned this one in. Turn this one in and report that I was the fellow who had found Morningstar and run away. The score would be low, very low. Marlowe, three murders. Marlowe practically knee-deep in dead men. And no reasonable, logical, friendly account of himself whatsoever (The High Window, op. cit. : 1140)

Je retourne dans le salon et je reste planté là, respirant la bouche ouverte et me demandant ce que ça donnerait, quand je déclarerais celui-là. Déclarer celui-là et reconnaître que je suis le gars qui a trouvé le cadavre de Morningstar et qui s'est débiné. Ça ne donnera pas grand-chose de bon... Ce sera même légèrement catastrophique... Marlowe : trois meurtres ; Marlowe, enfoncé jusqu'au cou dans des cadavres. Et pas de témoignage en sa faveur - raisonnablement, tout au moins (traduction personnelle).

\section{Conclusion}

En guise de conclusion, il convient de rappeler la grande originalité des romans de Chandler, qui, tout comme Conan Doyle, quelques décennies avant lui, fait office de pionnier dans le domaine du roman policier, voire de la narration, car les techniques du monologue intérieur et de l'introspection sont poussées à l'extrême, comme l'a révélé le large choix de citations proposé dans cet article. Outre le fait qu'elles éclairent indirectement le caractère du personnage de Philip Marlowe par ses réflexions et ses pensées, elles révèlent une vision peu bienveillante de la société de son temps. Même dans une période de soi-disant prospérité, il fait craquer le vernis du « Rêve américain ». Pour revenir à la citation insérée dans l'introduction, la " vérité dissimulée » qui constitue la quête principale du détective n'est-elle pas, justement, le besoin de révéler cette société jusque dans ses re- 
coins les plus cachés ? La question reste ouverte et, quoi qu'il en soit, le monologue intérieur se révèle un outil privilégié en ce sens ${ }^{6}$.

À tel point que, dans les adaptations des romans de Chandler sur grand écran, l'accent a constamment été mis sur la subjectivité et le fait que Marlowe reste le véritable narrateur de chaque enquête, tandis que l'utilisation des reflets souligne l'oscillation constante entre illusion et réalité. Dans Adieu, ma belle (Murder, My Sweet, 1944), adaptation d'Adieu, ma jolie, Marlowe est incarné par l'acteur Dick Powell. Au début du film, il est interrogé par la police et relate son enquête sur le mode de l'analepse. Ce choix donne plus de sens à la narration, car le spectateur sait à qui le détective s'adresse : après quelques plans d'introduction avec la ville de Los Angeles en toile de fond et la voix off de Marlowe, l'œil de la caméra nous montre le détective assis, le soir, dans le fauteuil de son bureau, avant que le personnage de Moose Malloy, son client, apparaisse d'abord dans le reflet de la vitre. Dans Le grand Sommeil (The Big Sleep, 1946), la plus célèbre des adaptations de Chandler sur grand écran - dont le scénario fut confié à William Faulkner -, Humphrey Bogart incarne Philip Marlowe et, détail plus que révélateur, il est présent à chaque scène et aucune péripétie ne se déroule sans lui. L'enquête avance ainsi au fil de ses actions et de ses interrogations. De cette omniprésence à une introspection encore plus poussée, il n'y avait qu'un pas et il fut franchi par l'acteur et metteur en scène Robert Montgomery dans La Dame du lac (Lady in the Lake, 1947). Cette œuvre se caractérise par une utilisation permanente de la « caméra subjective » et la technique narrative de Chandler y trouve son illustration la plus parfaite, puisque l'œil de la caméra se confond avec celui de Philip Marlowe excepté au début, alors que le détective s'adresse brièvement au spectateur pour présenter son enquête - et son visage n'apparaît que dans le reflet d'un miroir. ${ }^{7}$ Enfin, dans Adieu, ma jolie (Farewell, My Lovely, 1975), c'est Robert Mitchum qui endosse le rôle du célèbre détective et le film commence à nouveau par quelques plans généraux

6. Ce procédé a même été repris de l'autre côté de l'Atlantique, quelques années plus tard, par l'écrivain Léo Malet, qui, lui aussi, relate les aventures du détective privé Nestor Burma à la première personne du singulier.

7. Il est possible de regarder cette scène en cliquant sur le lien suivant : $<$ https://www.youtube.com/watch?v=Zzdl-js_mQ4> 
de Los Angeles, avant de présenter Marlowe. Il regarde par une fenêtre, tandis que l'enseigne lumineuse d'un hôtel s'y reflète et qu'il parle en voix off pour décrire la ville et son propre état d'esprit, dans un style rappelant celui de Chandler et le monologue intérieur :

"This past spring was the first that I felt tired and realized I was growing old. Maybe it was the rotten weather we'd had in L.A., maybe the rotten cases I'd had - mostly chasing a few missing husbands and then chasing their wives, once I found them, in order to get paid. Or maybe it was just the plain fact that I am tired and growing old ».

Au printemps dernier, je me suis senti fatigué pour la première fois et j'ai pris conscience que je vieillissais. Peut-être à cause du temps pourri que nous avions eu à Los Angeles, peut-être à cause des affaires minables dont j'avais eu à m'occuper. La plupart du temps, rechercher quelques maris disparus et, ensuite, rechercher leur épouse, une fois que je leur mettais le grappin dessus, afin d'être payé. Ou, à dire vrai, je suis peut-être fatigué et je commence à avoir l'âge de mes artères (traduction personnelle).

Babel, EA 2649

\section{Bibliographie}

ChANDLER Raymond (1995), Stories and Early Novels (dir. Frank MacShane), The Library of America, New York.

CHANDleR Raymond (1995), Later Novels and Other Writings (dir. Frank MacShane), The Library of America, New York.

DUJARDIN Edouard ([1931] 2001), « Le Monologue intérieur, son apparition, ses origines, sa place dans l'œuvre de James Joyce et dans le roman contemporain ", Les Lauriers sont coupés (dir. Jean-Pierre Bertrand). GF Flammarion, Paris.

\section{Filmographie}

Adieu, ma belle (Murder, My Sweet), dir. Edward Dmytryk. RKO Pictures, 1944.

Le grand Sommeil (The Big Sleep), dir. Howard Hawks. Warner Bros., 1946.

La Dame du lac (Lady in the Lake), dir. Robert Montgomery. MetroGoldwyn-Mayer, 1947.

Adieu, ma jolie (Farewell, My Lovely), dir. Dick Richards. Avco Embassy Pictures, 1975. 